\title{
Microwave Characterization of Metal-Decorated Carbon Nanopowders Using a Single Transmission Line
}

\author{
Francisco Mederos-Henry $\mathbb{D}^{1},{ }^{1}$ Sophie Hermans $\mathbb{D}^{1},{ }^{1}$ and Isabelle Huynen $\mathbb{D}^{2}$ \\ ${ }^{1}$ Institute of Condensed Matter and Nanosciences (IMCN), Université catholique de Louvain, 1348 Louvain-la-Neuve, Belgium \\ ${ }^{2}$ Institute of Information and Communications Technologies, Electronics and Applied Mathematics (ICTEAM), Université catholique \\ de Louvain, 1348 Louvain-la-Neuve, Belgium
}

Correspondence should be addressed to Isabelle Huynen; isabelle.huynen@uclouvain.be

Received 30 June 2018; Revised 14 November 2018; Accepted 4 December 2018; Published 18 April 2019

Academic Editor: Stefano Bellucci

Copyright $\odot 2019$ Francisco Mederos-Henry et al. This is an open access article distributed under the Creative Commons Attribution License, which permits unrestricted use, distribution, and reproduction in any medium, provided the original work is properly cited.

This paper presents a novel approach for the characterization of microwave properties of carbon-based nanopowders, decorated or not with magnetic nanoparticles. Their microwave parameters, dielectric constant, electrical conductivity, and complex magnetic permeability are extracted from measurements performed using a single transmission line acting as a test cell. Two geometries of transmission line were tested, and successful results were obtained with each one of them. The measurement results are assessed by a phenomenological model enabling to fit the measurement of the dielectric constant and conductivity, providing an insight on the compacity quality of the powder sample. Also, the extraction of the permeability is validated by the detection of a ferromagnetic resonance showing a linear dependence on external DC magnetic field.

\section{Introduction}

Microwave characterization of materials is of prime interest for a number of applications. The knowledge of the electromagnetic constitutive parameters (complex permittivity and the associated electrical conductivity and magnetic permeability) is mandatory to design a number of microwave devices including electromagnetic absorbers, attenuators, and resistors. Over the past years, various methods were proposed using different topologies. The quantities most often measured are the reflection and/or transmission coefficient of the microwave signal in free space in the presence of the material acting as a screen or a reflector, the resonant frequency and loaded $Q$ factor of a resonator containing a sample of the material under scope, and the measurement of the reflection and transmission coefficients at the end of a transmission line in which the material acts as a discontinuity. The test cell can be a waveguide, a coaxial line, or a planar topology. At microwaves and millimeter waves, the permittivity is derived from the measured variation of the reflection coefficient [1] at the input of a shortened waveguide [2] or a resonant cavity [3] loaded by a solid sample. For liquids or powders, transmission lines combining reflection and transmission measurements are preferred: they use waveguides or TEM (transverse electric magnetic) transmission lines, coaxial [4], or not $[5,6]$. Transmission line methods suffer from strong uncertainties when their length is multiples of half-wavelengths since transmission and reflection measurements [7] or reflection measurements only [8] are used for extracting permittivity and permeability.

A lot of work has been done in previous years by our team on the characterization of (nano)materials, but embedded in an alumina $[9,10]$ or polymer [11-13] template. We recently extended our studies to the characterization of nanopowders themselves $[14,15]$ and developed subsequently an efficient single-line method for their direct characterization, which is illustrated in this paper.

In order to achieve a broadband characterization, we exploit here a property of the line-return-line (LRL) method [16] that provides, as a by-product, the propagation constant of the line from which the permittivity $\varepsilon_{\mathrm{r}}$ is extracted [17]. Our formulation does not include reflection measurements and hence does not suffer from half-wave uncertainties. However, the propagation constant cannot discriminate 


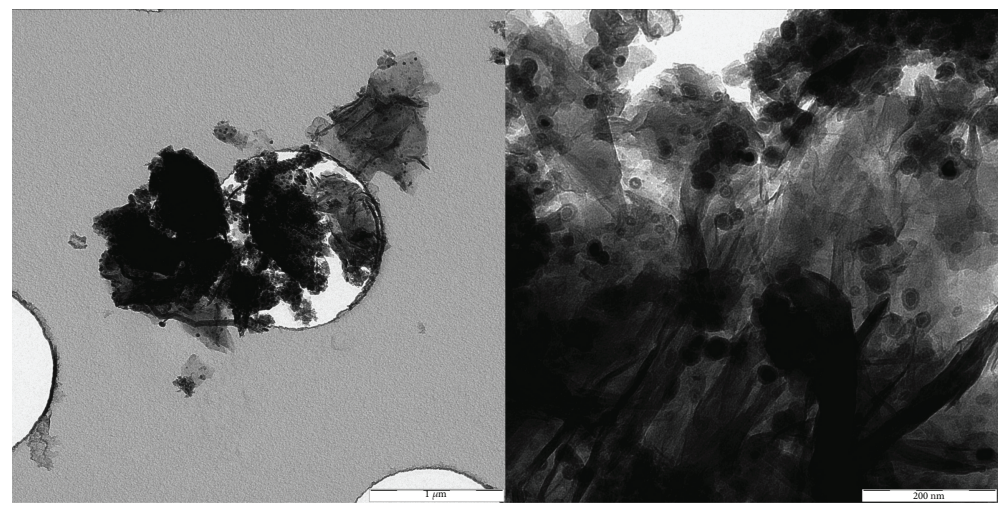

FIGURE 1: TEM images of graphene oxide decorated with zerovalent iron nanoparticles (ZVI@GO) at different magnifications.

between permittivity $\varepsilon_{\mathrm{r}}$ and permeability $\mu_{\mathrm{r}}$ since it involves only their product. Permittivity $\varepsilon_{\mathrm{r}}$ can be extracted for nonmagnetic materials only. Hence, we propose to use a single line, successively measured twice: once empty and once filled with the material of interest. This combination enables to retrieve the sample's $\varepsilon_{\mathrm{r}}$ and $\mu_{\mathrm{r}}$ parameters simultaneously, requiring the fabrication of a single line only.

The main originality of our work is that it allows us to extract the parameters of the sole nanopowder. In the literature, nanostructures are usually dispersed in an insulating polymer [18-22] or wax [23-25] matrix and only the microwave properties of the whole nanocomposite mixture are retrieved. We study here decorated carbon-based nanopowders preferable to other powders precisely because there are no methods reported for their characterization in the microwave frequency range, and as part of a research project "Nano4waves" [26] aimed at developing carbon nanotubes (CNTs) and graphene nanoplatelets (GNPs) decorated with magnetic nanoparticles (MNPs) for the design of devices enabling the control of electromagnetic (EM) wave propagation.

Another originality of our method is to make the retrieval of $\varepsilon_{\mathrm{r}}$ and $\mu_{\mathrm{r}}$ possible by exploiting two different features. Firstly, the permittivity $\varepsilon_{\mathrm{r}}$ is extracted by combining the empty line measurement with the measurement of the filled line under a high-DC magnetic field. This ensures that the permeability of the material is equal to unity during the measurement. Secondly, the permeability $\mu_{\mathrm{r}}$ is extracted from the combination of the measurement of the filled line under zero-DC magnetic field and the prior measurement of the empty line.

Section 2 (Materials and Methods) presents the synthesis of the nanopowders, the fabrication of the two transmission lines used for their microwave measurement, and the characterisation tools. Section 3 (Results) presents successively the extraction procedure for permittivity and permeability based on measurements of the empty and filled lines, the phenomenological model for obtaining the complex permittivity of the nanopowder proposed in [11], which provides information on the compacity of the powder, and finally, the permittivity and permeability extracted from the measurement of the empty and filled lines. Measured permittivity $\varepsilon_{\mathrm{r}}$ is assessed by the phenomenological model. Measured permeability $\mu_{\mathrm{r}}$ is assessed by the detection of a ferromagnetic resonance (FMR) following a linear dependence on external applied DC magnetic field and by a phenomenological model of the permeability. Section 4 concludes about the main features of this work.

\section{Materials and Methods}

2.1. Synthesis of Nanopowders. This section describes the preparation of the nanocomposite powders under scope.

2.1.1. Pristine Nanocarbon Supports (NcS). Three types of graphenic nanocarbon supports $(\mathrm{NcS})$ were employed: graphene oxide (GO), reduced graphene oxide (rGO) (both from Nanoinnova Technologies SL, Spain), and graphene nanoplatelets (GNPs; TIMCAL, Switzerland). These NcS were microwave characterized as received.

2.1.2. Decoration of Graphene Oxide (GO) with Magnetic Nanoparticles (MNPs). Metallic zerovalent iron (ZVI) and nickel (Ni) nanoparticles (NPs) were deposited onto graphene oxide (GO). The synthetic methodologies employed will be briefly described below. In both cases, the engaged quantities aimed a 50\% metal/GO weight loading rate (LR). Corresponding TEM pictures are given in Figures 1 and 2.

2.1.3. Synthesis of ZVI@GO Nanocomposite. The preparation of ZVI nanoparticles supported on GO was performed using a Pechini-type sol-gel method. In a typical synthesis, $50 \mathrm{mg}$ of graphene oxide (GO), $180.8 \mathrm{mg}$ of iron (III) nitrate nonahydrate $\left(\left(\mathrm{FeNO}_{3} \bullet 9 \mathrm{H}_{2} \mathrm{O}\right) 99+\%\right.$, Across $), 516 \mathrm{mg}$ of citric acid (CA; analytical grade, Merck), and a magnetic stir bar were introduced in a small round-bottom flask. $3.3 \mathrm{ml}$ of ethanol (TechniSolv, VWR) was added, the flask was closed with a septum, and the mixture was stirred until complete dissolution of the reactants. The suspension was then sonicated at $80 \mathrm{~W}$ for 1 hour (VWR ultrasound cleaner USC 1200THD) in order to disperse GO. $112 \mu$ l of ethylene glycol (EG; spectrophotometric grade, Alfa Aesar) was added, and the suspension was heated with a reflux condenser in an oil bath at $90^{\circ} \mathrm{C}$ for 3 hours. The suspension was then poured in a porcelain combustion boat and placed in a drying oven under vacuum at $30^{\circ} \mathrm{C}$ for 2 hours. The obtained gel-covered ceramic plate was introduced in a tubular furnace (Carbolite Gero STF16, United Kingdom) and heated to $750^{\circ} \mathrm{C}$ for $2 \mathrm{~h}$ under $\mathrm{Ar} / \mathrm{H}_{2} \quad 95 / 5$ atmosphere. The 


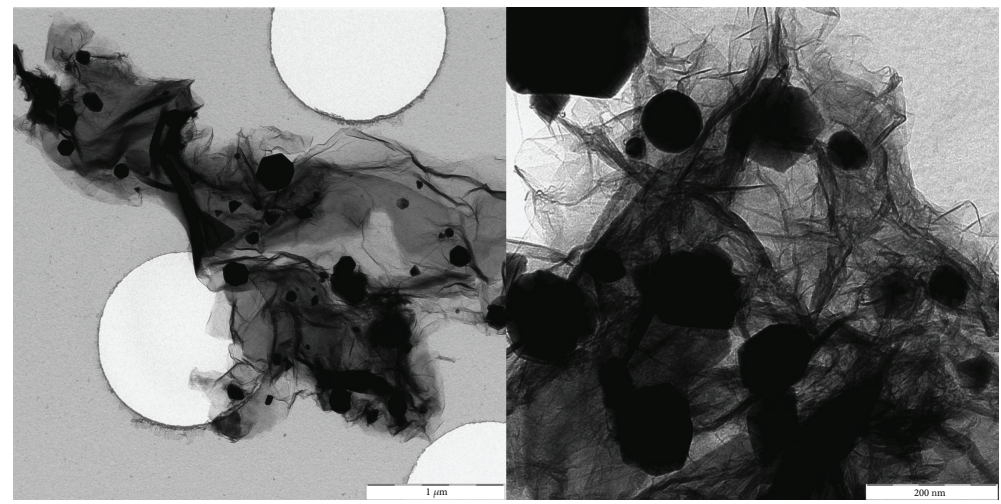

FIGURE 2: TEM images of graphene oxide decorated with nickel nanoparticles (Ni@GO) at different magnifications.

temperature was increased and decreased at a rate of $1.7^{\circ} \mathrm{C} / \mathrm{min}$. The recovered powder was stored in air.

2.1.4. Synthesis of Ni@GO Nanocomposite. GO was decorated with nickel nanoparticles using a one-pot solvothermal method. In a typical synthesis, $50 \mathrm{mg}$ of GO was dispersed in $40 \mathrm{ml}$ of ethylene glycol (EG) and sonicated for 120 minutes in a VWR ultrasound cleaner USC 1200-THD at $80 \mathrm{~W}$ sonication power. $110 \mathrm{mg}$ of nickel (II) acetylacetonate $\left(\mathrm{Ni}(\mathrm{acac})_{2}, 95 \%\right.$, Sigma-Aldrich) was then added to the above suspension under vigorous stirring. Subsequently, an aqueous $1 \mathrm{M} \mathrm{NaOH}$ (Normapur, VWR) solution was added dropwise, under vigorous stirring, until a $\mathrm{pH}$ value of 10.5 was reached. After stirring for 45 minutes, the mixture was transferred into a $300 \mathrm{ml}$ stainless steel autoclave vessel with a Teflon lining and heated at $190^{\circ} \mathrm{C}$ for 24 hours. The autoclave temperature was automatically regulated by a Parr 4836 temperature controller (Parr Instrument Company, USA) having an average heating rate of $4^{\circ} \mathrm{C} / \mathrm{min}$. The autoclave inner atmosphere was never purged or modified in any manner during the synthesis, while the system pressure was left to build up during heating without any type of external control. Reaction solutions were continuously stirred at $300 \mathrm{rpm}$ while heating. When reaction time was completed, the autoclave was immediately immersed in an ice bath and cooled down to ambient temperature. Reaction products were separated from the reaction media by magnetic decantation and then recovered by filtration over a PVDF filter (Millipore GVWP02500, 0.22 $\mu \mathrm{m}$ pore size). Afterwards, they were purified by rinsing six times, alternating Milli-Q water and ethanol. Finally, they were dried under vacuum at room temperature for 12 hours.

\subsection{Characterization}

2.2.1. Transmission Electron Microscopy (TEM). Samples were dispersed in hexane by sonication. Three drops of the supernatant were then deposited onto a holey carbon film supported on a copper grid (C-flat, Protochips, USA), and left to dry, overnight, at room temperature under vacuum. TEM images were obtained on a LEO 922 OMEGA Energy Filter Transmission Electron Microscope operating at $120 \mathrm{kV}$.

2.2.2. Fabrication of the Transmission Line Test Cells. Two geometries of transmission lines (TLs) were tested: a

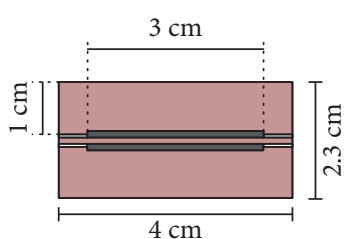

(a)

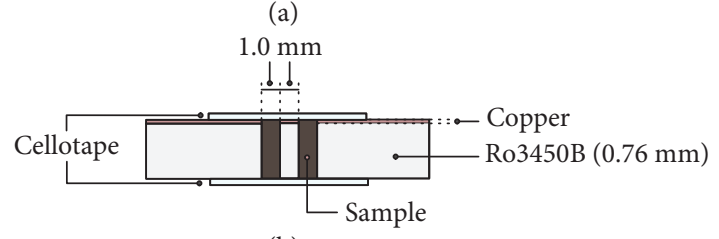

(b)

FIgURE 3: Dimensions of the coplanar waveguide (CPW) line for measurements (not in scale). (a) top view and (b) side view. The Teflon substrate is represented in light grey whereas the top copper layer is colored in dark pink and the nanopowder is black.

coplanar waveguide (CPW) and a microstrip. The CPW device consisted of a $0.762 \mathrm{~mm}$ thick Teflon substrate of type Ro4350B onto which a $17 \mu \mathrm{m}$ thick copper layer was deposited. By using an Oxford J-1064/355 pulsed laser system, two parallel lines were etched lengthwise on the copper substrate, exposing the Teflon substrate. For this etching procedure, the $355 \mathrm{~nm}$ laser was chosen, operated at $0.5 \mathrm{~W}$, with a speed of $200 \mathrm{~mm} / \mathrm{s}$ and a frequency of $200 \mathrm{kHz}$, pulsing every 10 ps for 11 cycles. Leaving the line extremities untouched, their central section was fully etched through the thickness of the substrate by using the $1064 \mathrm{~nm}$ laser at $1.6 \mathrm{~W}$ during four cycles, while the speed, frequency, and pulse length were kept constant. More details about the dimensions of the obtained device can be found in Figure 3. The same procedure applies for the microstrip. However, a two-substrate configuration was used: cavities were drilled in the top Ro3010 substrate, stripped of its copper layers, in order to prevent short circuit between the microstrip and ground in cases of highly conductive nanopowders. The bottom layer was machined out of a Ro4003 substrate for which only the bottom $17 \mu \mathrm{m}$ thick copper layer was conserved (see Figure 4).

In both cases, the same method was employed to fill the TL. The nanopowders were mixed with ethanol in order to form a slurry that could be introduced into the TLs' 


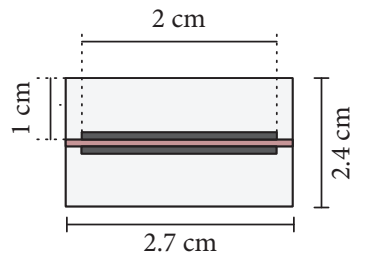

(a)

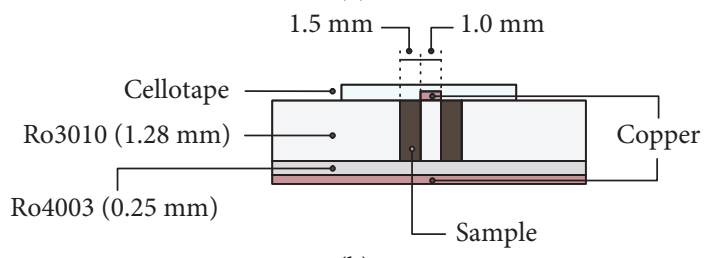

(b)

FiguRE 4: Geometry of the microstrip line for measurements (not in scale). (a) top view and (b) side view. The Teflon substrates are represented in light grey and grey white whereas the strip and ground copper layers are colored in dark pink, the cellotape in light blue, and the nanopowder in black.

rectangular cavities. A Teflon spatula was then used to compact the samples. The procedure was repeated thrice in order to compactly fill the cavities. Afterwards, the as-filled TLs were dried overnight under vacuum, at ambient temperature, to eliminate the solvent. Finally, nanopowder leftovers were removed with a thin cotton swab impregnated with ethanol in order to clean the TL surface. After filling, a piece of cellotape was placed on top of the microstrip substrate in order to maintain the low-density nanopowders in the cavities. In the case of the CPW substrate, cellotape was placed before and after filling on the bottom and top sides, respectively. Tests were performed to verify that the cellotape had no effect on the TL line electromagnetic characteristics. It has to be emphasized that the same microstrip (or CPW) line was used for all nanopowders characterized in this paper. Cavities were simply carefully cleaned using ethanol before moving from one powder sample to another.

2.2.3. Scattering Matrix (S-Parameters) Measurements. The microwave response of the empty and sample-filled microstrip and coplanar waveguide lines (CPW) was measured under DC magnetic field applied perpendicularly to the substrate using a NTM 10400M-260 electromagnet supplying magnetic field values ranging from 0 to 9 kOersteds (kOe). The samples were placed in the gap of the electromagnet and connected to one end of a set of long coaxial cables using a pair of Anritsu $36801 \mathrm{~K}$ right angle launchers. The other coaxial cables ends were connected to the ports of a 12-term calibrated Agilent N5245A PNA-X 70GHz vector network analyzer (VNA), placed away from the electromagnet's intense DC magnetic field. Data acquisition of the transmission $S$-parameters was performed using LabVIEW.

\section{Results and Discussion}

In this section, we will present the extraction procedure for the characterization of nanopowders. It is important to stress here that the extraction of permittivity and
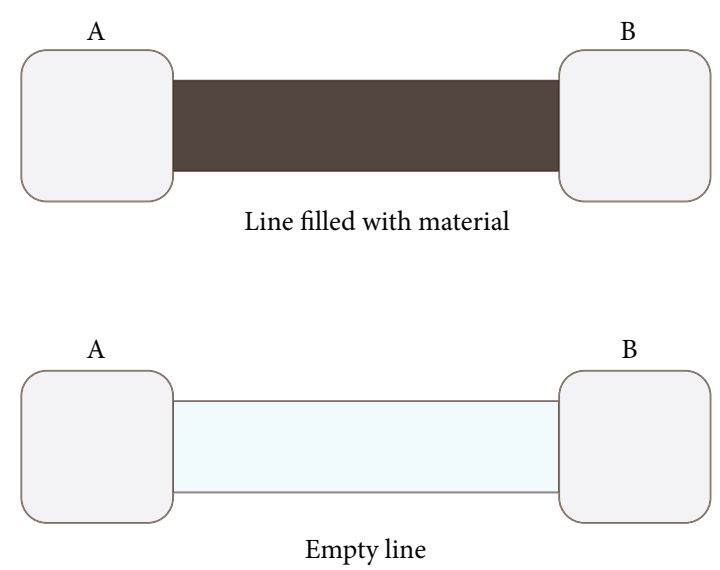

FIGURE 5: Schematic of the measurement configuration.

permeability is based on the propagation constant that takes into account the whole cross-section of the TL used, including the surrounding air visible in Figures 3 and 4. As a consequence, the next section will first present the extraction method for the so-called effective permeability and permittivity of each sample, denoted by subscript "e", taking into account the surrounding air. Afterwards, the formulas for the retrieval of relative parameters of the characterized nanopowders themselves, denoted by subscript "r", will be presented.

3.1. Extraction Procedure for Permittivity and Permeability. Figure 5 shows a schematic view of the measurement configuration, adapted from [10]. Boxes A and B hold for the transmission of signal between the source of VNA and input of transmission line (A) and from the output of transmission line to detector of VNA (B). They include cables from the source to the connectors and the connectors from the cables to the transmission lines. Their transmission factors are noted as $S_{21 \mathrm{~A}}$ and $S_{21 \mathrm{~B}}$, respectively. The transmission factor of the transmission line having length $L$ is expressed as $e^{-\gamma L}$, where $\gamma$ is the propagation constant of the line. The extraction of permittivity and permeability is based on the successive measurement of the filled line under zero-DC magnetic field, of the filled line under high-DC magnetic field, and of the empty line. The transmission factor from the source to the detector for filled line under zero-DC magnetic field can be expressed as the product of the three transmission factors as follows:

$$
S_{21 \text { filled }}=S_{21 \mathrm{~A}} e^{-\gamma \text { filled } L} S_{21 \mathrm{~B}},
$$

and for empty lines, it writes

$$
S_{21 \text { empty }}=S_{21 \mathrm{~A}} e^{-\gamma \text { empty } L} S_{21 \mathrm{~B}}
$$

where $\gamma_{\mathrm{m}}=j 2 \pi f \sqrt{\varepsilon_{\mathrm{em}} \mu_{\mathrm{em}}} / c_{o}$. In these equations, e denotes that these are the effective parameters, $m$ indicates if the transmission line is filled or empty, $L$ is the length of the line, $c_{o}$ is the light velocity in air, and $f$ is the frequency of the signal. The material contribution to the measurement is thus 

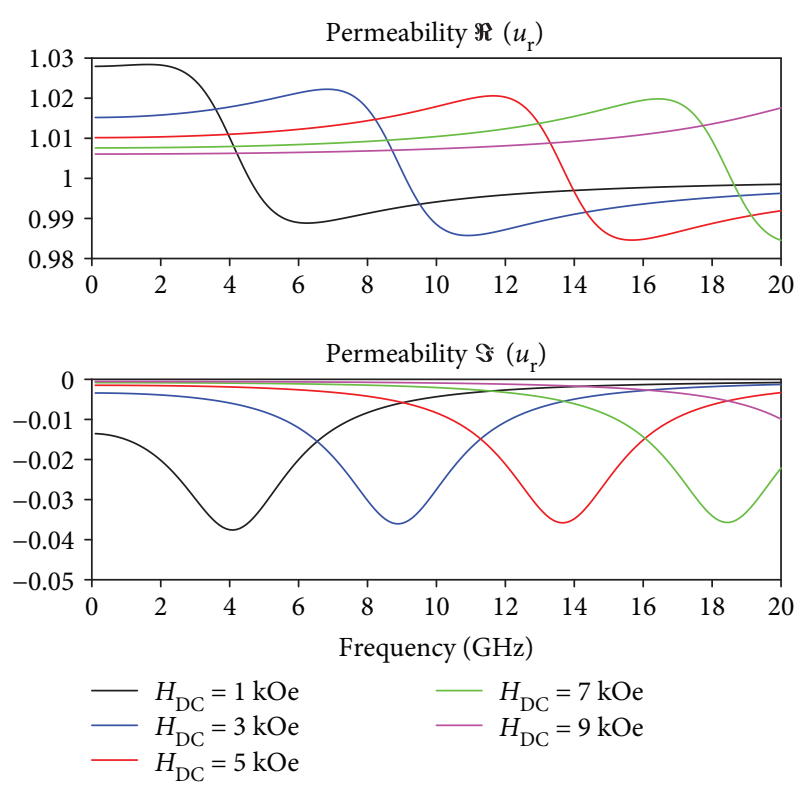

Figure 6: Permeability calculated according to (12) with $\alpha=0.082$, $4 \pi M_{\mathrm{s}}=720$ Oe, and $\tau=8 \times 10^{-11}$.

included in the propagation constant $\gamma_{\mathrm{m}}$ via $\mu_{\mathrm{e}}$ and $\varepsilon_{\mathrm{e}}$. Using these equations, we can derive from the ratio $S_{21 \text { filled }} / S_{21 e m p t y}$ :

$$
\begin{aligned}
\frac{\gamma_{\text {filled }}-\gamma_{\text {empty }}}{2 \pi f L / c_{o}} & =\frac{-\ln \left(S_{21 \text { filled }} / S_{21 \text { empty }}\right)}{2 \pi f L / c_{o}} \\
& =j\left(\sqrt{\varepsilon_{\text {efilled }} \times \mu_{\text {efilled }}}-\sqrt{\varepsilon_{\text {eempty }} \times \mu_{\text {eempty }}}\right) .
\end{aligned}
$$

As $\varepsilon_{\text {eempty }}=\mu_{\text {eempty }}=1$ since the empty line is filled with air, we use another measurement performed on the filled line in order to extract $\varepsilon_{\mathrm{e}}$. A high magnetic field is applied during the frequency measurement of the filled line in order to move any magnetic effect above the frequency range under scope resulting into $\mu_{\text {efilled }} \rightarrow 1$, yielding

$$
\begin{aligned}
\frac{\gamma_{\text {field }}-\gamma_{\text {empty }}}{2 \pi f L / c_{o}} & =\frac{-\ln \left(S_{21 \text { field }} / S_{21 \text { empty }}\right)}{2 \pi f L / c_{o}} \\
& =j \sqrt{\varepsilon_{\text {efilled }}}-1,
\end{aligned}
$$

since $\gamma_{\text {field }}=\sqrt{\varepsilon_{\text {efilled }}}\left(2 \pi f L / c_{o}\right)$. The high magnetic field considered in this work is equal to 9 kOersteds. As shown in Figure 6, this value ensures that the permeability tends to 1 for the magnetic nanopowders studied in this paper. It corresponds also to the magnetic saturation of the samples as measured in $[27,28]$. From these last two equations, the permittivity and the permeability of the material are extracted. The permittivity $\varepsilon_{\mathrm{e}}$, defined as the permittivity extracted from the filled line $\varepsilon_{\text {efilled }}$, is obtained from (4), using the measurement under high magnetic field ensuring that $\mu_{\text {efilled }}=1$ so that only $\varepsilon_{\mathrm{e}}$ is involved and can be retrieved. Then, the permeability $\mu_{\mathrm{e}}$ defined as the permeability $\mu_{\text {efilled }}$ extracted

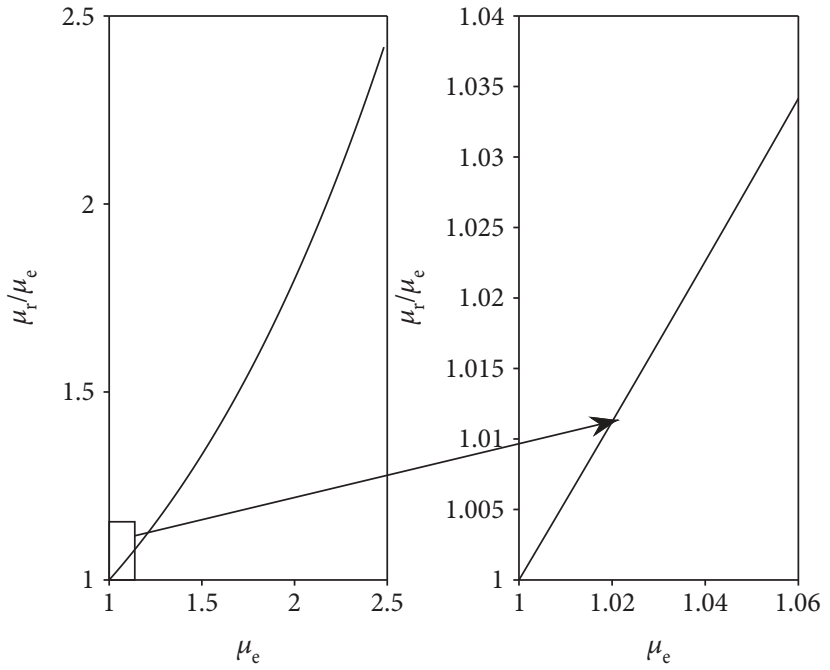

Figure 7: Ratio of relative by effective permeability $\mu_{\mathrm{r}} / \mu_{\mathrm{e}}$ for the microstrip topology of Figure 3, calculated from an equation in [30] with $T=1.53 \mathrm{~mm}$ and $W_{\mathrm{s}}=1.00 \mathrm{~mm}$.

from the filled line is obtained by introducing the permittivity $\varepsilon_{\mathrm{e}}$ in (3). The procedure is also applied in this paper for measuring permeabilities as a function of an applied DC magnetic field of a given value $H$. In this case, three measurements are considered: empty line, filled line under the $H$ field, and filled line under the $9 \mathrm{kOe}$ field.

For the microstrip topology of Figure 4, the permittivity $\varepsilon_{\text {s }}$ of the substrate supporting the microstrip and hosting the filled cavities is retrieved from the effective one $\varepsilon_{\mathrm{e}}$ using the following expression derived from [29]:

$$
\varepsilon_{\mathrm{s}}=2 \times \frac{\varepsilon_{\mathrm{e}}-1+F}{1+F},
$$

with

$$
F=\frac{1}{\sqrt{1+12 H / W_{S}}}+0.04\left\{1-\left(W_{S} / T\right)^{2}\right\} \text {, }
$$

where $T$ is the total thickness of the substrate and $W_{\mathrm{s}}$ the width of the microstrip. For the topology of Figure $4, F=$ 0.231 . The relative permeability of the whole substrate including the filled cavities is obtained from the following chart in Figure 7 derived from expressions in [30]. The relative values that will be presented in Section 3.3.2 are close to 1. This is due, firstly, to the low density and dusty nature of the material under scope and, secondly, to the low loading rate of MNPs on GO nanoplatelets. As a consequence, the intrinsic permeability of the powder grains is diluted in the surrounding air inside the cavity of the test cell. Thus, in the current experiment, effective permeability values obtained from (3) and (4) are close to 1.02. Using Figure 7, it can be found that the corrected substrate permeability value would thus be equal to $1.011 \times 1.02=1.0312$. This slight difference represents less than a $2 \%$ correction.

For the CPW line topology of Figure 3, the derivation of substrate values from the effective ones is much simpler since 
the following well-known assumption holds [31]:

$$
\begin{aligned}
& \varepsilon_{\mathrm{s}}=2 \varepsilon_{\mathrm{e}}-1, \\
& \mu_{\mathrm{s}}=2 \mu_{\mathrm{e}}-1 .
\end{aligned}
$$

Finally, we are able to retrieve the contribution of the sole nanopowder by using a simple volumetric factor taking into account the volume occupied by the nanopowder in the cavities as compared to the total volume present around the central strip conductor. If the width of each cavity is noted at $W$, and the width of the central strip is noted at $W_{s}$, then the parameters of the sole nanopowder are obtained from those extracted from (3) and (4) using the multiplicative factor $V$ :

$$
V=\frac{2 W+W_{s}}{2 W}
$$

Factor $V$ compensates the volumetric dilution of the powder in the whole substrate.

To summarize, the full procedure for the retrieval of the parameters $\varepsilon_{\mathrm{r}}$ and $\mu_{\mathrm{r}}$ of the nanopowder follows these three steps:

(i) The effective parameters $\varepsilon_{\mathrm{e}}$ and $\mu_{\mathrm{e}}$ of the transmission line whose cavities are filled with the nanopowder are obtained from the measured $S$-parameters of the lines empty, filled, and filled under $9 \mathrm{kOe}$ DC magnetic field, using equations (3) and (4)

(ii) The parameters $\varepsilon_{\mathrm{s}}$ and $\mu_{\mathrm{s}}$ of the inhomogeneous substrate are obtained from $\varepsilon_{\mathrm{e}}$ and $\mu_{\mathrm{e}}$ using equation (5) and Figure 7 in the case of microstrip line and equations (7) and (8) for the case of the CPW line

(iii) The parameters $\varepsilon_{\mathrm{r}}$ and $\mu_{\mathrm{r}}$ of the nanopowder are obtained by multiplying the substrate parameters by the factor $V$, i.e.,

$$
\begin{aligned}
\varepsilon_{\mathrm{r}} & =V \varepsilon_{\mathrm{s}}, \\
\mu_{\mathrm{r}} & =V \mu_{\mathrm{s}} .
\end{aligned}
$$

In order to assess the quality of the extraction method, we have simulated the $S$-parameters of the empty, filled, and under $9 \mathrm{kOe}$ microstrip and CPW lines shown in Figures 3 and 4 using COMSOL Multiphysics simulation software, for a material in the cell having nominal predefined values of $\varepsilon_{\mathrm{r}}$ and $\mu_{\mathrm{r}}$ of the powder. From the simulated $S$-parameters of the microstrip configuration, we used equation (5) and Figure 7 to retrieve the permeability and permittivity values. The retrieved values given in Table 1 are in close agreement with the predefined values for the sample inserted in the cell for the simulation. We did the same for the CPW configuration and with the same predefined values. Again, a good agreement is observed.

3.2. Phenomenological Model for Complex Permittivity. In order to assess the efficiency of the method, extracted permittivity $\varepsilon_{\mathrm{r}}$ will be compared to a model previously developed for
TABLE 1: Nominal (bold) and retrieved (italics) values of $\varepsilon_{\mathrm{r}}, \mu_{\mathrm{r}}$, and $\sigma_{\mathrm{r}}$, using $S$-parameters of microstrip and CPW topologies of Figures 3 and 4 simulated with COMSOL Multiphysics and equations (3), (4), (5), (7), (8), (9), and (10).

\begin{tabular}{lcccc}
\hline Case & Topology & $\varepsilon_{\mathrm{r}}$ & $\sigma_{\mathrm{r}}(\mathrm{S} / \mathrm{m})$ & $\mu_{\mathrm{r}}$ \\
\hline 1 & Microstrip & $\mathbf{3 . 0} 2.971$ & $\mathbf{0 . 5} 0.4934$ & $\mathbf{1 . 5} 1.501$ \\
2 & Microstrip & $\mathbf{6 . 0} 6.07$ & $\mathbf{1 . 0} 0.92$ & $\mathbf{3 . 0} 3.14$ \\
3 & CPW & $\mathbf{3 . 0} 2.97$ & $\mathbf{0 . 5} 0.44$ & $\mathbf{1 . 5} 1.497$ \\
4 & CPW & $\mathbf{6 . 0} 6.18$ & $\mathbf{1 . 5} 1.26$ & $\mathbf{3 . 0} 3.1$ \\
\hline
\end{tabular}

conductive inclusions dispersed in an insulating matrix [11], illustrated in Figure 8. In the present paper, conductive inclusions are the grains of the powder, surrounded by insulating air, as a whole forming the nanopowder. According to the model in [11], the random distribution of NPs is approximated by a network of grains, separated by air. Each grain is assumed to be conductive, having an equivalent resistor, and strongly coupled to its neighbors by a capacitor. The resulting model is a 2-layered transmission line. The equivalent complex permittivity $\varepsilon_{\mathrm{p}}$ of the nanopowder has the following expression:

$$
\frac{1}{\varepsilon_{\mathrm{p}}} \triangleq \frac{1}{\left(\varepsilon_{\mathrm{r}}+\sigma / j \omega \varepsilon_{o}\right)}=\frac{T_{2}+T_{1} /\left[\varepsilon_{\mathrm{NPs}}+\sigma_{\mathrm{NPs}} / j \omega \varepsilon_{o}\right]}{T_{1}+T_{2}},
$$

where $\varepsilon_{\mathrm{NPs}}$ is the dielectric constant of the NPs and $\sigma_{\mathrm{NPs}}$ their conductivity, $\varepsilon_{o}$ is the permittivity of vacuum, and $T_{i}$ are the different layer thicknesses.

The inputs for the phenomenological model (11) are as follows: the dielectric constant of the nanoparticles under scope, their intrinsic conductivity, while fill represents the filling factor defined as the ratio: fill $=T_{2} /\left(T_{1}+T_{2}\right)$, which models the proximity between NPs inducing capacitive coupling responsible for the variation in frequency of the dielectric constant and conductivity of the nanocomposite agglomerate. When the phenomenological model is used to fit the measurements, values of fill give an insight on the compacity of the nanopowder, hence on the reproducibility of the filling method of the cavities.

3.3. Determination of Microwave Properties of Nanopowders. In this section, we present the extraction of parameters for nonmagnetic and magnetic conductive nanopowders characterized with the techniques described in Sections 2.2.3 and 3.1. Figures 9-12 show the results obtained for the pristine nondecorated nanocarbon supports, reduced graphene oxide (rGO), and graphene nanoplatelets (GNPs), as well as for the ZVI@GO and Ni@GO synthetized nanocomposites. These results are discussed hereafter.

3.3.1. Pristine NcS Powders. Figure 9 shows the dielectric constant and conductivity extracted from the measurement of reduced graphene oxide ( $\mathrm{rGO}$ ) filling the microstrip line and of the same microstrip kept empty. Figure 10 shows the same for graphene nanoplatelets (GNPs). The conductivity reached at high frequency for rGO is lower than that for GNPs, as expected for such oxidized and defective 


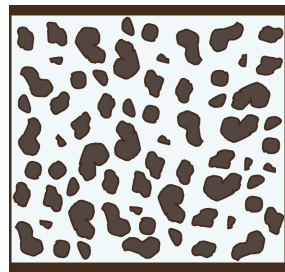

(a)

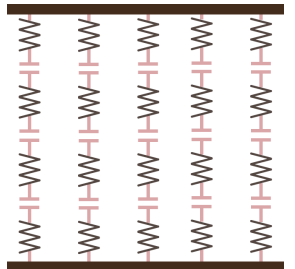

(b)

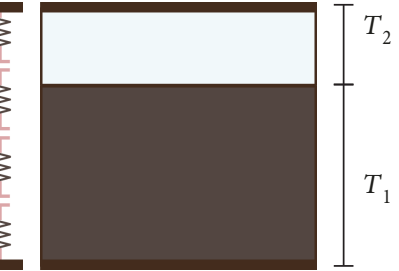

(c)

Figure 8: Schematic representation of model. (a) Topology of a nanocomposite, (b) equivalent circuit, and (c) two-layered equivalent transmission line.
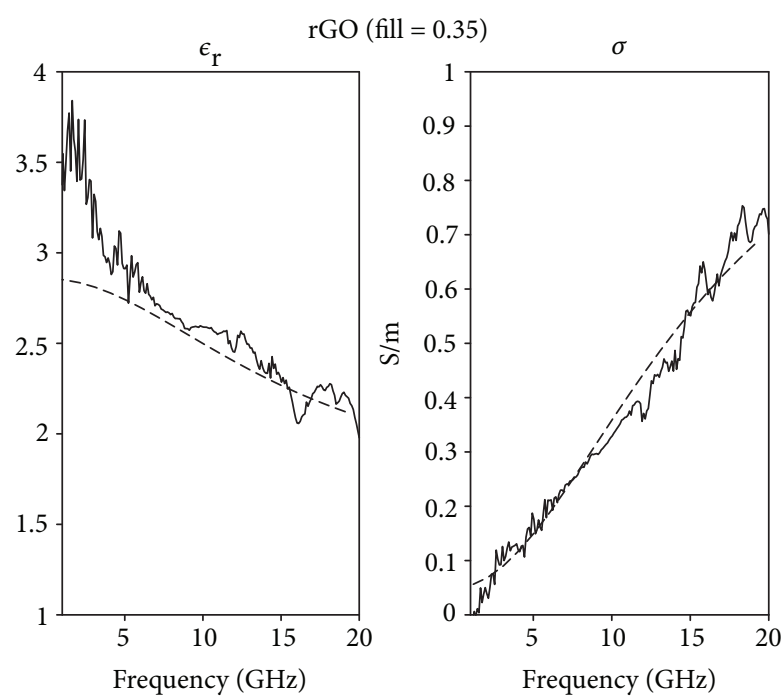

Figure 9: Dielectric constant and conductivity extracted from (3), (4), and (5) for rGO nanopowder. Solid line shows extraction from measurement; dashed line shows phenomenological prediction from (10).
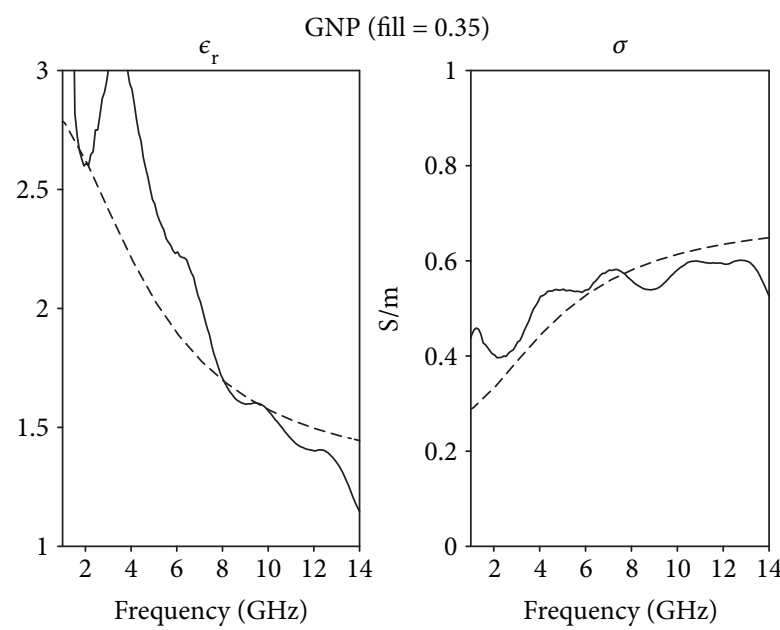

FIGURE 10: Dielectric constant and conductivity extracted from (3), (4), and (5) for GNP nanopowder. Solid line shows extraction from measurement; dashed line shows phenomenological prediction from (10).

nanocarbon supports. The filling factor is similar for the two kinds of nanoparticles (0.35). Since typical volumetric densities of GNPs and GO are reported to be similar $\left(1.85 \mathrm{~g} / \mathrm{cm}^{3}\right.$

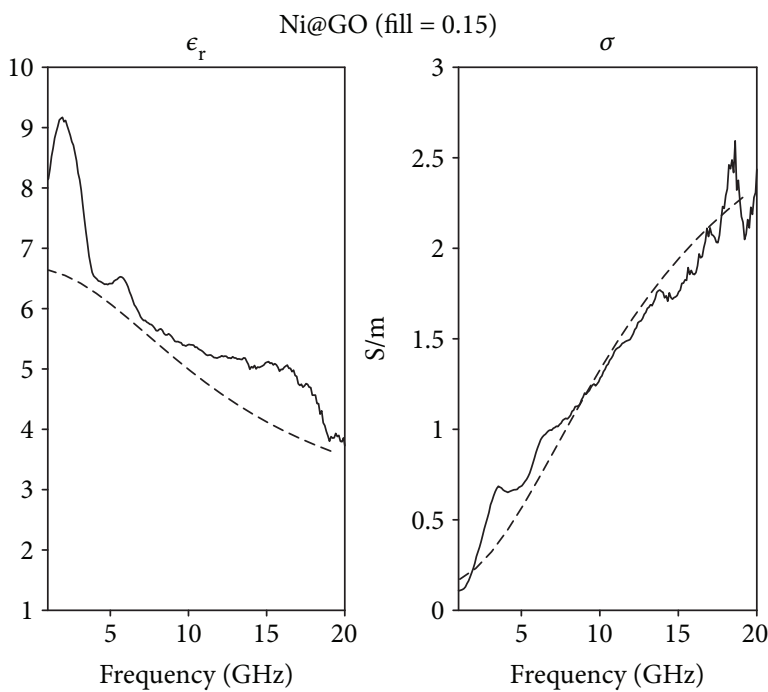

FIGURE 11: Dielectric constant and conductivity extracted from (3), (4), and (6) for Ni@GO nanopowder. Solid line shows extraction from measurement; dashed line shows phenomenological prediction from (10).

versus $\left.1.9 \mathrm{~g} / \mathrm{cm}^{3},[32,33]\right)$, this result suggests that the same amount of nanopowders is introduced into the cavities and that the filling of the cavities is thus reproducible.

It is worth mentioning here that oscillation ripple observed in the experimental curves is due to the residual mismatch between the $50 \Omega$ reference impedance of the VNA measuring equipment and the characteristic impedance of the MS and CPW lines used for the characterization [34]. The value of the latter impedance depends on the dielectric constant and permeability of the supporting substrate, hence also on the parameters $\varepsilon_{\mathrm{r}}$ and $\mu_{\mathrm{r}}$ of the included nanopowder. As they are a priori unknown, the best possible strategy is to design the geometry of the line kept empty, in order to achieve $50 \Omega$. When filled lines are measured, their mismatch against $50 \Omega$ induced by the filling powder creates the well-known ripple phenomenon [34] on the $S$-parameters, and so, on $\varepsilon_{\mathrm{r}}$ and $\mu_{\mathrm{r}}$, as they are deduced from $S$-parameters using (3) and (4). Despite this ripple, the trend behavior of $\varepsilon_{\mathrm{r}}$ and $\mu_{\mathrm{r}}$ is clearly retrieved from their mean value and further assessed by the phenomenological model (11).

Also, we have checked the reproducibility of our procedure by making 5 measurements spread over 8 months on a same sample. Observed variation on the extracted permittivity is less than $3 \%$. This illustrates the good repeatability 

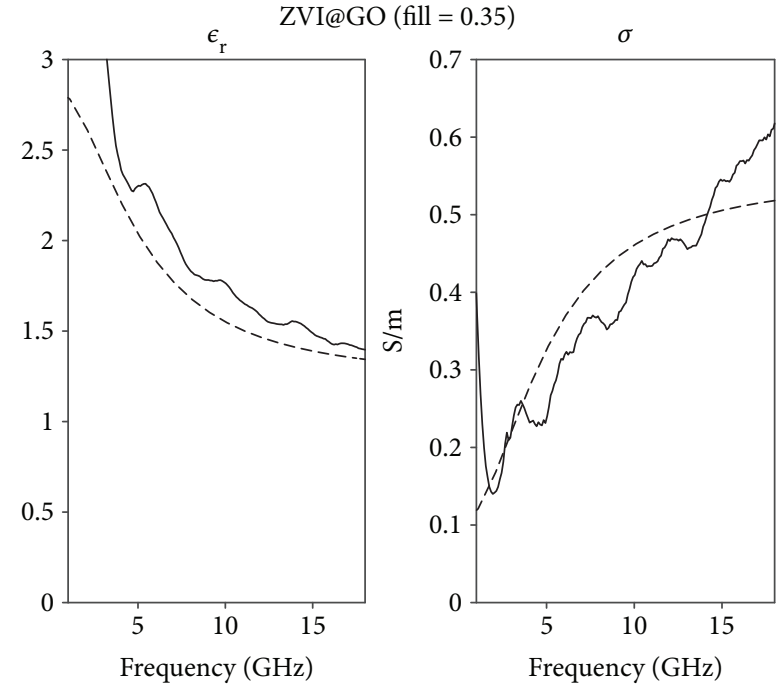

FIGURE 12: Dielectric constant and conductivity extracted from (3), (4), and (6) for ZVI@GO nanopowder. Solid line shows extraction from measurement; dashed line shows phenomenological prediction from (10).

of the extraction procedure and the excellent stability of our samples over time.

As stated in the Introduction, there is no other method published in the scientific literature that allows to directly characterize the EM properties of nanopowders without prior sample preparation. The closest-matching methodology loads the nanopowder into a paraffin matrix, and a toroidal-shape test sample is produced from this mixture [23-25]. Reported amounts of loaded nanopowders typically range from 130 to $220 \mathrm{mg}$. While with our methodology, the $\mathrm{CPW}$ and microstrip line cavities require approximately 1.1 and $1.9 \mathrm{mg}$ to be filled, respectively. The reported amounts, almost 100 -fold superior to those used in our test cells, give an indication on the acute sensitivity of our methodology.

3.3.2. Ferromagnetic Conductive Powders. Figures 11 and 12 show the conductivity and dielectric constant extracted from the measurement of the two ferromagnetic samples, nickel $(\mathrm{Ni})$ and zerovalent iron (ZVI) nanoparticles, deposited onto graphene oxide (GO). As previously mentioned, the method implies using two sets of measurements: one in which the $\mathrm{CPW}$ line is filled with each nanopowder and the other in which it is kept empty. As can be observed in both figures, the phenomenological model successfully fits the extracted conductivity and dielectric constant over the whole frequency range.

Figures 13 and 14 show the extracted complex permeability $\mu_{\mathrm{r}}$. The measurement is made for several values of DC magnetic field applied perpendicularly to the line substrate. The imaginary part shows a minimum (marked "O") depending on the DC field which is responsible for the so-called ferromagnetic resonance (FMR) [35] absorption. For each sample, the validity of the extraction of $\mu_{\mathrm{r}}$ can be assessed by drawing the dispersion relation showing the FMR frequency (where the minimum of $\mathbb{I}=\left(\mu_{\mathrm{r}}\right)$ occurs) as a function of applied DC magnetic field. Figures 15 and 16
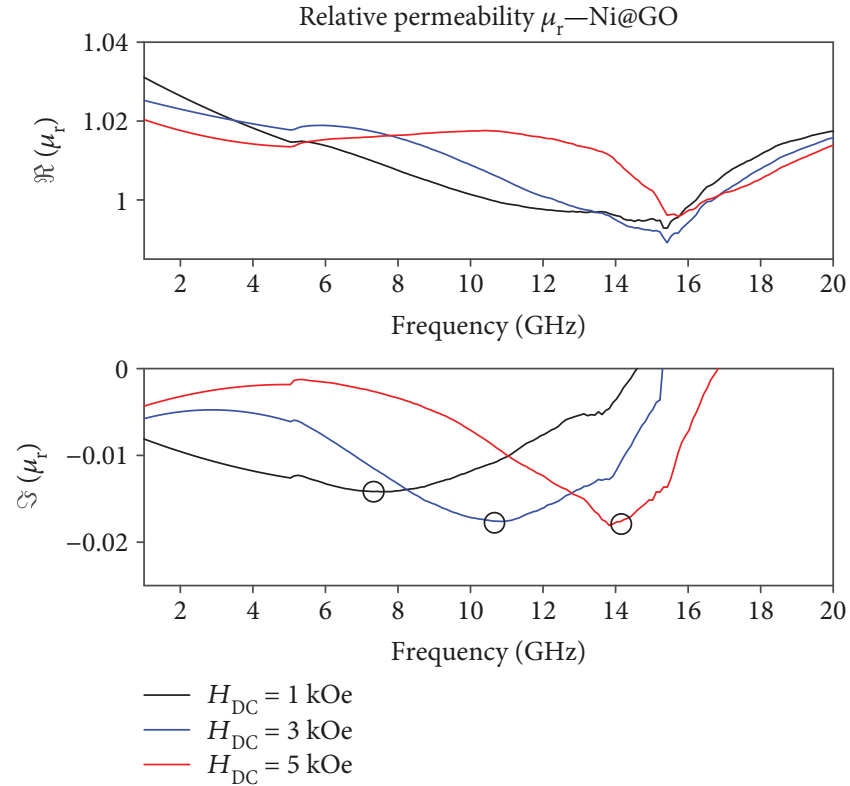

Figure 13: Permeability extracted from (3), (4), and (7) for Ni@GO nanopowder. Circles indicate the location of the minima corresponding to FMR frequency.
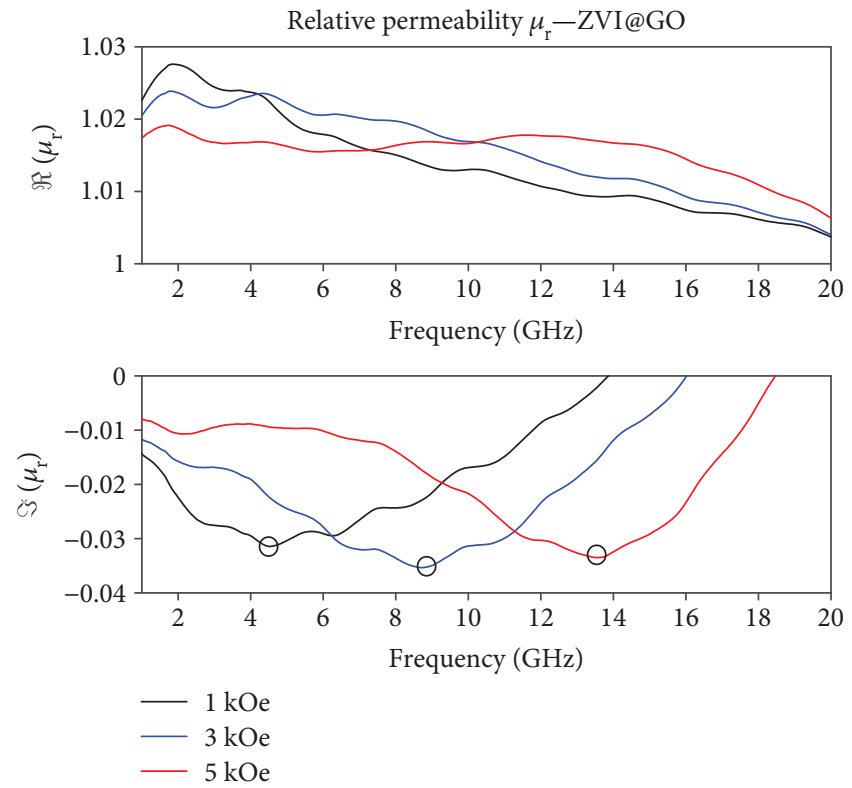

FIgURE 14: Permeability extracted from (3), (4), and (7) for ZVI@GO nanopowder. Circles indicate the location of the minima corresponding to FMR frequency.

show for each sample a linear relation in accordance with the theoretical law for FMR:

$$
f_{\mathrm{FMR}}=g\left\{H_{\mathrm{DC}}+H_{\mathrm{a}}\right\}
$$

where $g$ is the gyromagnetic factor equal to $2.8 \mathrm{MHz} / \mathrm{Oe}$ and $H_{\mathrm{a}}$ is the effective anisotropy field proportional to the saturation magnetization of the ferromagnetic nanoparticles. 


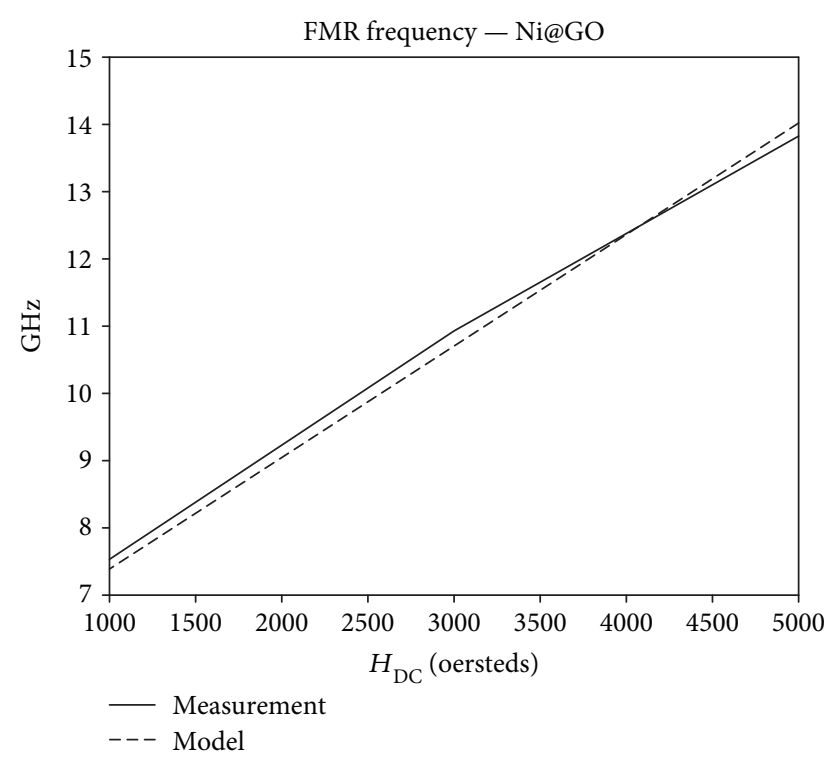

FIGURE 15: FMR dispersion relation for Ni@GO nanopowder, extracted from Figure 13 (solid) and calculated according to (11) (dashed) with $H_{\mathrm{a}}=24.4$ Oe.

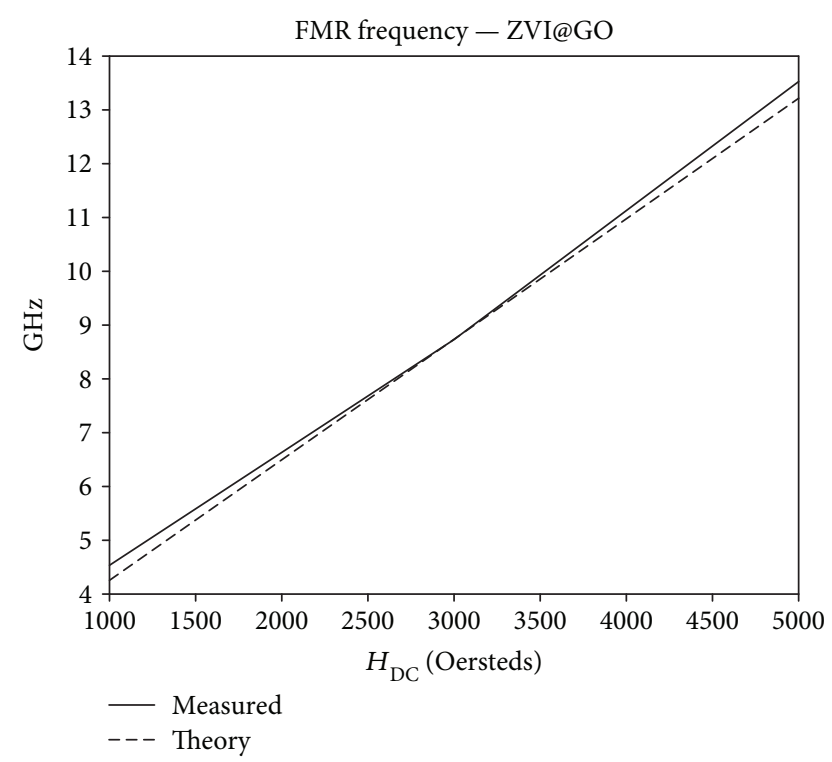

FIGURE 16: FMR dispersion relation for ZVI@GO nanopowder, extracted from Figure 14 (solid) and calculated according to (11) (dashed) with $H_{\mathrm{a}}=720$ Oe.

As another assessment, the permeability of our ferromagnetic material can be modelled by a FMR formalism derived from [35]. Its expressions is

$$
\mu_{\mathrm{r}}=1+\frac{\alpha 4 \pi M_{\mathrm{s}} g f_{\mathrm{r}}}{\left(f_{\mathrm{r}}+j / \tau\right)^{2}-f^{2}} .
$$

In these equations, $f_{\mathrm{r}}=g\left[\mathrm{H}_{\mathrm{a}}+H_{\mathrm{DC}}\right]$ is the ferromagnetic resonance (FMR) frequency, $\tau$ is the relaxation time associated to the damping factor, $f$ is the RF frequency, $\alpha$ holds for the dilution of MNPs in the nanopowder, and $M_{\mathrm{s}}$ and $g$ are the saturation magnetization and the gyromagnetic ratio, respectively. Figure 6 shows the simulation of the permeability for different values of the applied DC magnetic field. It is observed that at 9 , the complex permeability tends towards unity, since the FMR resonance vanishes. This means that for this value, equation (4) holds. The simulation shows a good agreement with measurements of ZVI@GO shown in Figure 14.

\section{Conclusion}

In this paper, we have demonstrated the efficiency of a method based on a single transmission line reusable for all samples to extract both complex permittivity and magnetic permeability of low density nanocomposite powders. We present a novel method for the extraction of dielectric constant and conductivity, which is successfully validated by implementing a phenomenological model. This model includes, as input parameters, the dielectric constant and conductivity of nanoparticles and a filling factor modelling the distance between particles. In consequence, it also enables to assess the quality of the filling of the cavities with nanopowders. The magnetic permeability is also extracted and modelled successfully and further assessed by an accurate retrieval of the FMR frequency that closely follows the linear theoretical law. This highlights that the procedure is efficient for this kind of low density samples. Although the proposed extraction method used here is for nanopowders, it can also be applied to other topologies of lines and other materials such as ferrites, liquids, or ferrofluids, provided they can easily be inserted in the cavities.

This is of prime interest for the design of numerous microwave devices such as absorbers, filters, and metamaterials.

\section{Data Availability}

The data used to support the findings of this study are included within the article.

\section{Conflicts of Interest}

The authors declare no conflicts of interest regarding this publication.

\section{Acknowledgments}

The authors thank the participants to the Nano4waves project for fruitful discussions, Mr. Rajkumar Jaiswar for the design of the microstrip line, and Mr. Tommy Haynes for performing the TEM imagery. The authors are grateful to the National Fund for Scientific Research (Fonds De La Recherche Scientifique - FNRS, Belgium) for supporting this research. This work is also supported by the Walloon region and by the "Communaute Française de Belgique", through the project "Nano4waves" funded by its research program "Actions de Recherche Concertees." 


\section{References}

[1] M. Sucher and J. Fox, Handbook of Microwave Measurements, vol. 2, John Wiley \& Sons Inc, 3rd edition, 1963.

[2] G. Kent, "A dielectrometer for the measurement of substrate permittivity," Microwave Journal, vol. 34, pp. 72-82, 1991.

[3] M. Olyphant, "Microwave permittivity measurements using disk cavity specimens," IEEE Transactions on Instrumentation and Measurement, vol. IM-20, no. 4, pp. 342-344, 1971.

[4] J. Huang, K. Wu, P. Morin, and C. Akyel, "Characterization of highly dispersive materials using composite coaxial cells: electromagnetic analysis and wideband measurement," IEEE Transactions on Microwave Theory and Techniques, vol. 44, no. 5, pp. 770-777, 1996.

[5] K.-H. Baek, H.-Y. Sung, and W. S. Park, "A 3-position transmission/reflection method for measuring the permittivity of low loss materials," IEEE Microwave and Guided Wave Letters, vol. 5, no. 1, pp. 3-5, 1995.

[6] J. L. Medina, A. Serrano, and F. J. Mendieta, "Microstrip effective dielectric constant measurement and test of CAD models up to $20 \mathrm{GHz}$," Microwave Journal, vol. 36, pp. 82-993, 1993.

[7] E. Ni, "An uncertainty analysis for the measurement of intrinsic properties of materials by the combined transmissionreflection method," IEEE Transactions on Instrumentation and Measurement, vol. 41, no. 4, pp. 495-499, 1992.

[8] H. B. Sequeira, "Extracting $\varepsilon$ and $\mu$ of materials from vector reflection measurements," Microwave Journal, vol. 34, pp. 73-85, 1991.

[9] A. Encinas-Oropesa, M. Demand, L. Piraux, U. Ebels, and I. Huynen, "Effect of dipolar interactions on the ferromagnetic resonance properties in arrays of magnetic nanowires," Journal of Applied Physics, vol. 89, no. 11, pp. 6704-6706, 2001.

[10] S. Pignard, G. Goglio, I. Huynen, A. Radulescu, and L. Piraux, "Ferromagnetic resonance in metallic nanowires," in INTERMAG 2000 Digest of Technical Papers. 2000 IEEE International Magnetics Conference, pp. 3482-3484, Royal York Hotel Toronto, ON, Canada, April 2000.

[11] A. Saib, L. Bednarz, R. Daussin et al., "Carbon nanotube composites for broadband microwave absorbing materials," IEEE Transactions on Microwave Theory and Techniques, vol. 54, no. 6, Part II, pp. 2745-2754, 2006.

[12] I. Molenberg, M. Planckaert, L. Jonckheere, E. Ferain, and I. Huynen, "Investigation of ionic conductivity in tracketched nanoporous polyimide membranes using a microwave technique," Microwave and Optical Technology Letters, vol. 53, no. 9, pp. 2060-2063, 2011.

[13] I. Molenberg, M. M. Bernal, P. Bollen et al., "Simple, convenient, and non-destructive electromagnetic characterization technique for composite and multiscale hybrid samples at microwave frequencies," Microwave and Optical Technology Letters, vol. 56, no. 2, pp. 504-509, 2014.

[14] F. Mederos-Henry, B. P. Pichon, Y. T. Yagang et al., "Decoration of nanocarbon solids with magnetite nanoparticles: towards microwave metamaterial absorbers," Journal of Materials Chemistry C, vol. 4, no. 15, pp. 3290-3303, 2016.

[15] F. Mederos-Henry, S. Hermans, and I. Huynen, "Coplanar waveguide method for microwave and ferromagnetic resonance characterization of nanocarbon powders decorated with magnetic nanoparticles," Microwave and Optical Technology Letters, vol. 59, no. 9, pp. 2330-2335, 2017.
[16] H. J. Eul and B. Schiek, "A generalized theory and new calibration procedures for network analyzer self-calibration," IEEE Transactions on Microwave Theory and Techniques, vol. 39, no. 4, pp. 724-731, 1991.

[17] I. Huynen, C. Steukers, and F. Duhamel, "A wideband line-line dielectrometric method for liquids, soils, and planar substrates," IEEE Transactions on Instrumentation and Measurement, vol. 50, no. 5, pp. 1343-1348, 2001.

[18] Z. Wang, J. Luo, and G.-. L. Zhao, "Dielectric and microwave attenuation properties of graphene nanoplatelet-epoxy composites," AIP Advances, vol. 4, no. 1, article 017139, 2014.

[19] O. A. Al-Hartomy, A. A. Al-Ghamdi, F. Al-Salamy et al., "Dielectric and microwave properties of graphene nanoplatelets/carbon black filled natural rubber composites," International Journal of Materials and Chemistry, vol. 2, no. 3, pp. 116-122, 2012.

[20] O. A. Al-Hartomy, A. Al-Ghamdi, F. Al-Salamy et al., "Effect of carbon nanotubes and graphene nanoplatelets on the dielectric and microwave properties of natural rubber composites," Advanced Composite Materials, vol. 22, no. 5, pp. 361-376, 2013.

[21] O. A. Al-Hartomy, A. A. Al-Ghamdi, N. Dishovsky, R. Shtarkova, V. Iliev, and F. El-Tantawy, "Comparative study of the influence of carbon nanotubes and graphene nanoplatelets on dielectric and microwave properties of conductive rubber composites," Research and Reviews in Polymer, vol. 3, no. 3, pp. 107-116, 2012.

[22] T. Zhao, C. Hou, H. Zhang et al., "Electromagnetic wave absorbing properties of amorphous carbon nanotubes," Scientific Reports, vol. 4, no. 1, article 5619, 2014.

[23] Y. Zhan, F. Meng, Y. Lei, R. Zhao, J. Zhong, and X. Liu, "One-pot solvothermal synthesis of sandwich-like graphene nanosheets/Fe3O4 hybrid material and its microwave electromagnetic properties," Materials Letters, vol. 65, no. 11, pp. 1737-1740, 2011.

[24] P. Liu, Y. Huang, and X. Zhang, "Synthesis, characterization and excellent electromagnetic wave absorption properties of graphene/poly (3,4-ethylenedioxythiophene) hybrid materials with $\mathrm{Fe}_{3} \mathrm{O}_{4}$ nanoparticles," Journal of Alloys and Compounds, vol. 617, pp. 511-517, 2014.

[25] P. Liu, Y. Huang, and X. Zhang, "Superparamagnetic $\mathrm{Fe}_{3} \mathrm{O}_{4}$ nanoparticles on graphene-polyaniline: Synthesis, characterization and their excellent electromagnetic absorption properties," Journal of Alloys and Compounds, vol. 596, pp. 25-31, 2014.

[26] Y. Danlee, R. Jaiswar, F. Mederos Henry et al., "Nano4Waves: a metamaterial approach towards smart nanocomposites for nanosecond signal control," in 2015 IEEE 15th International Conference on Nanotechnology (IEEE-NANO), pp. 188-191, Rome, Italy, 2015.

[27] F. Mederos-Henry, S. Depaifve, A. Wolf et al., "Thin microwave absorber films based on multi-walled carbon nanotubes solvothermally decorated with nickel nanoparticles".

[28] F. Mederos-Henry, J. Mahin, B. P. Pichon et al., "Novel microwave absorbers based on zero-valent $\mathrm{Fe}-\mathrm{Fe}_{2} \mathrm{O}_{3}$ and $\mathrm{Fe} / \mathrm{Co} / \mathrm{Ni}$ carbon-protected alloy nanoparticles supported on reduced graphene oxide".

[29] D. Packiaraj, K. J. Vinoy, and A. T. Kalghatgi, “Approximate synthesis formulas for microstrip line with aperture in ground plane," International Journal of RF and Microwave ComputerAided Engineering, vol. 22, no. 1, pp. 124-130, 2012. 
[30] K. Yeo and M. Lancaster, "New model for the effective permeability of ferrite microstrip," in 4th IEEE High Frequency Postgraduate Student Colloquium, The University of Leeds, pp. 105-110, Leeds, UK, 1999.

[31] J. Laskar, S. Chakraborty, and A.-V. Pham, Advanced Integrated Communication Microsystems, Wiley, IEEE Press, 2009.

[32] http://www.matweb.com/search/datasheettext.aspx?matguid= 8dc6fcb328fc49b8853d0b56bb258199.

[33] https://www.graphenea.com/products/reduced-grapheneoxide-1-gram.

[34] J. P. Dunsmore, Handbook of Microwave Component Measurements: With Advanced VNA Techniques, Wiley, 2012.

[35] C. Kittel, "On the theory of ferromagnetic resonance absorption,” Physical Review, vol. 73, no. 2, pp. 155-161, 1948. 


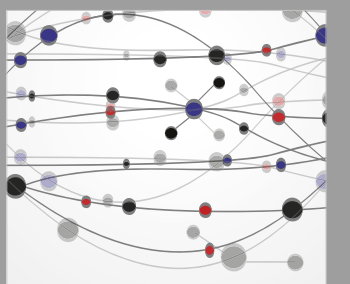

The Scientific World Journal
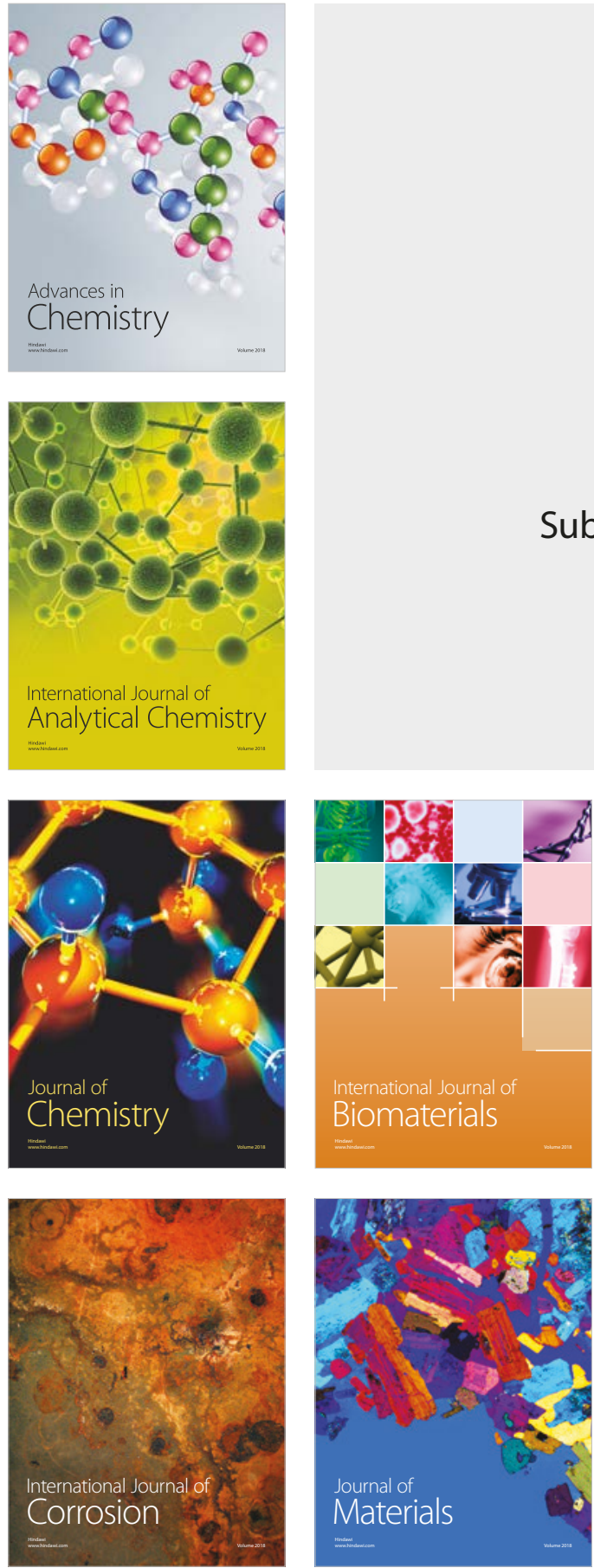

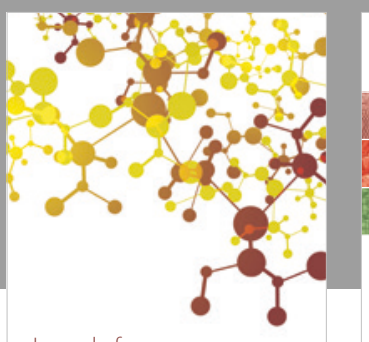

Journal of

Applied Chemistry
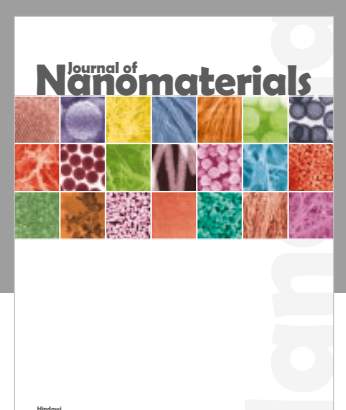

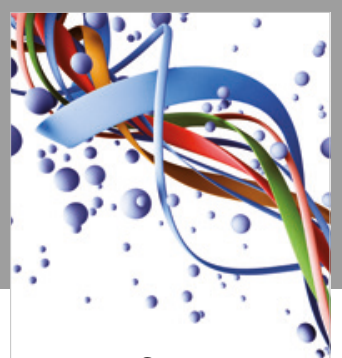

Scientifica

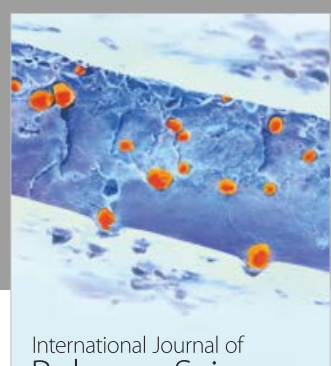

Polymer Science

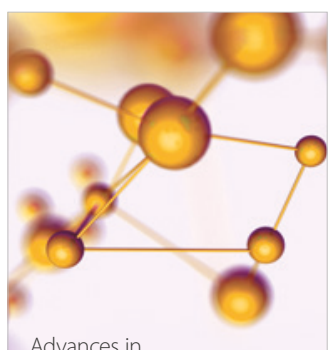

Physical Chemistry
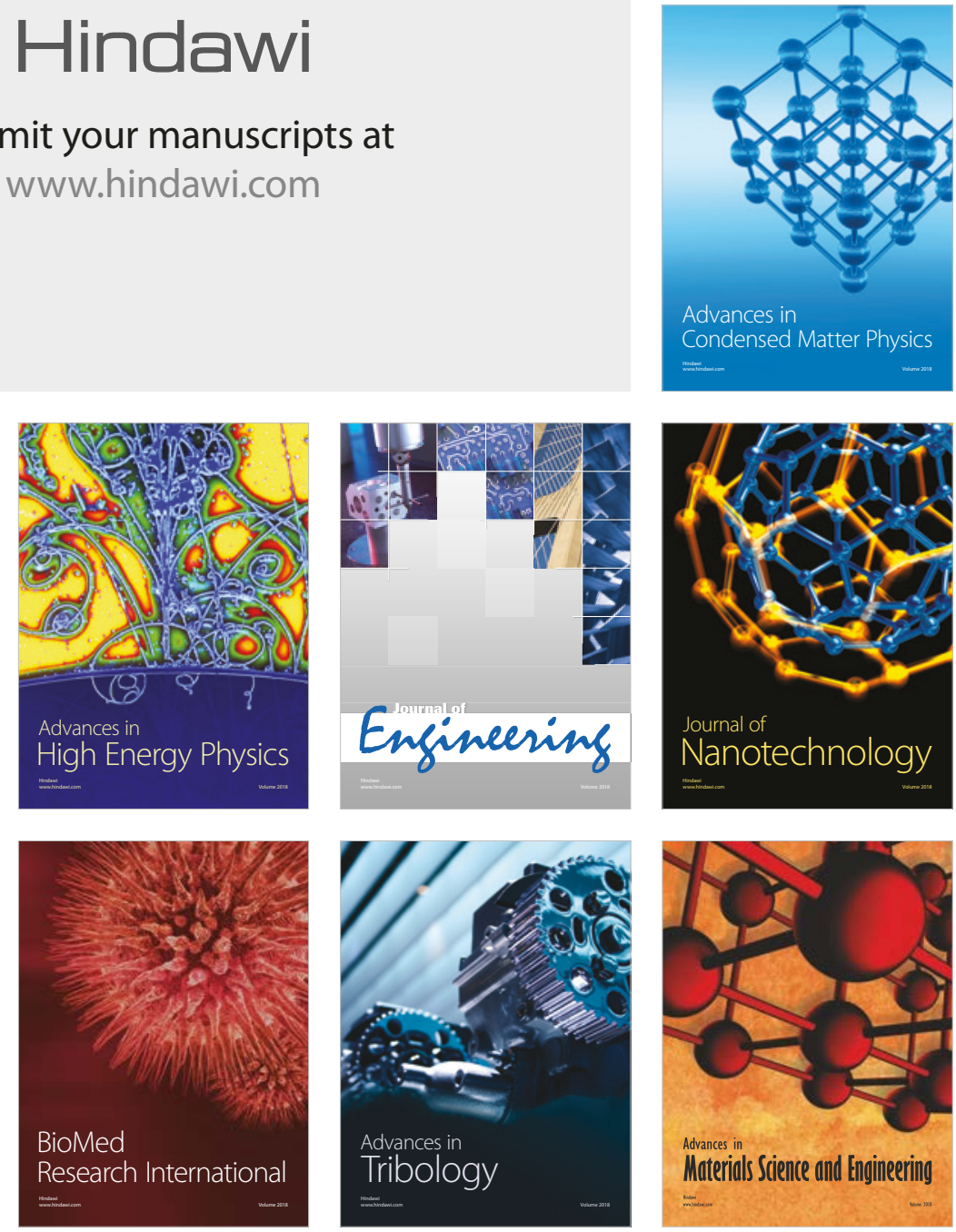